\title{
Prevalência de fraturas em cães e gatos atendidos em projeto de extensão da clínica cirúrgica na Cidade de Petrolina/PE - 2016 a 2018
}

Prevalence of fractures in dogs and cats assisted in an extension project of the surgical clinic in the

City of Petrolina/PE - 2016 a 2018

Prevalencia de fracturas en perros y gatos atendidos en un proyecto de ampliación de la clínica quirúrgica de la Ciudad de Petrolina/PR - 2016 a 2018

\section{Resumo}

As afecções ortopédicas são bastante comuns na medicina veterinária de pequenos animais, entre elas podemos destacar as fraturas. Objetivou-se verificar a prevalência de fraturas nos animais atendidos no Hospital Veterinário Universitário (HVU) da Universidade Federal do Vale do São Francisco (UNIVASF) pelo setor de Clínica Cirúrgica de Animais de Companhia (CCAC) em um projeto de extensão de atendimento especializado em clínica cirúrgica, e a partir deste levantamento, identificar os tipos de fraturas mais frequentes, os ossos mais acometidos, a influência da idade dos animais nas fraturas e as causas mais comuns destas. Para a realização deste estudo foi necessário o acesso aos prontuários e arquivos radiográficos do Hospital Veterinário Universitário da UNIVASF, nos quais compreendiam arquivos entres os anos de 2016 a 2018, totalizando 55 casos de fraturas, principalmente em esqueleto apendicular onde $87 \%$ foram em cães e $17 \%$ em gatos. Essas fraturas são bastante comuns no HVU - UNIVASF devido ao alto número de animais errantes e a falta de cuidado e orientação de alguns tutores na Cidade de Petrolina - Pernambuco.

Palavras chave: Trauma; Ortopedia; Cirurgia veterinária; Radiografia.

\begin{abstract}
Orthopedic conditions are quite common in animal veterinary medicine, which can be highlighted as fractures. The objective of this study was to verify the prevalence of fractures in the animals treated at the University Veterinary Hospital (HVU) of the Federal University of Vale do São Francisco (UNIVASF) by the Animal Surgery Clinic of the Company (CCAC) in an extension project of specialized care in surgical clnic and from this survey, fractures the most affected animals, the influence of infants on fractures and the most common causes of fractures. For an evaluation of this study, it was necessary to have access to the medical records and radiography of the University Veterinary Hospital of UNIVASF, which included data from 2016 to 2018, totaling 55 cases of fractures, mainly in medical records and $87 \%$ of dogs. These fractures are quite common in HVU - UNIVASF due to high numbers of wrong animals and the lack of care and guidance of some tutors in the City of Petrolina - Pernambuco.
\end{abstract}

Keywords: Trauma; Orthopedics; Veterinary surgery; Radiography. 


\begin{abstract}
Resumen
Las afecciones ortopédicas son bastante habituales en la medicina veterinaria para pequeños animales, entre las que podemos destacar las fracturas. El objetivo de este estudio fue verificar la prevalencia de fracturas en animales tratados en el Hospital Universitario Veterinario (HVU) de la Universidad Federal de Vale do São Francisco (UNIVASF) por la Clínica Quirúrgica de Animales de Compañía (CCAC) en un proyecto de extensión de atención especializada en clínica quirúrgica, y a partir de esta encuesta, identificar los tipos de fracturas más frecuentes, los huesos más afectados, la influencia de la edad de los animales en las fracturas y las causas más comunes de las mismas. Para la realización de este estudio fue necesario acceder a las historias clínicas y expedientes radiográficos del Hospital Veterinario Universitario de UNIVASF, que comprendieron expedientes entre los años 2016 a 2018, totalizando 55 casos de fracturas, principalmente en esqueleto apendicular donde el $87 \%$ estaban en perros y $17 \%$ en gatos. Estas fracturas son bastante comunes en HVU - UNIVASF debido a la gran cantidad de animales callejeros y la falta de cuidado y orientación de algunos tutores en la Ciudad de Petrolina - Pernambuco.
\end{abstract}

Palabras clave: Trauma; Ortopedia; Cirugía veterinaria; Radiografía.

\title{
1. Introdução
}

Em Medicina Veterinária de animais de companhia as afecções ortopédicas têm se tornado casos bastante comuns. Entre elas destacam-se as fraturas em ossos longos, especialmente o fêmur, que representa aproximadamente a metade de todas as fraturas nos ossos longos em cães e gatos (Beale, 2004; Decamp \& Schaefer, 2015; Harasen, 2003a). As fraturas obliquas são as mais registradas nesses ossos, enquanto que as fraturas cominutivas aparecem principalmente em fêmur e tíbia (Harasen, 2003b).

As fraturas oblíquas são fraturas causadas por cargas compressivas que geram estresse compressivo em direção axial e estresse de tração em direção circunferencial. Logo os estresses somados, geram linhas de fraturas que formam um ângulo com a linha perpendicular do eixo longo do osso. As fraturas oblíquas podem ser classificadas como oblíquas curtas (de $45^{\circ}$ ou menos de angulação) ou oblíquas longas (acima de $45^{\circ}$ de angulação) em relação ao eixo longo do osso (Fossum, 2019; Johnson \& Tobias, 2017).

Fraturas Cominutivas apresentam várias linhas de fraturas. Podem ser divididas em cominutivas simples moderadas ou severamente cominutivas. Podem sem compostas de três fragmentos, formando o padrão em borboleta ou em cunha. Ou fraturas de altamente cominutivas com mais de cinco fragmentos (Fossum, 2019).

Fraturas, são causadas, em sua maioria, por acidentes automobilísticos, mas também por projéteis balísticos, brigas e quedas (Fossum, 2019; Kumar et al., 2007; Johnson \& Olmstead, 1995). Cerca de 80\% da incidência de fraturas, deve-se à acidentes automobilísticos, e em $60 \%$ destas, os animais apresentam politraumatismo (Bonath,1998).

Além de um minucioso exame ortopédico o diagnóstico por imagem proporciona informações valiosas que indicam com mais precisão, o local, o tipo, a complexidade e demais complicações associadas às fraturas. O diagnóstico por imagem é uma das modalidades que ajuda na definição da melhor técnica de redução e estabilização de uma fratura (Thrall, 2014).

Ainda não há um estudo dedicado a prevalência de fraturas na cidade de Petrolina-PE. Logo este estudo tem como objetivo realizar um levantamento retrospectivo sobre a prevalência de fraturas em cães e gatos. E a partir desse levantamento caracterizar a espécie mais acometida, os ossos mais atingidos, a influência da idade e as causas mais comuns das fraturas em cães e gatos atendidos no HVU - UNIVASF na cidade de Petrolina - PE no período de 2016 a 2018.

\section{Metodologia}

Este artigo é um estudo de caráter quantitavio , que de acordo com Pereira et al. (2018) caracteriza-se pela coleta de dados numéricos, os quais dão origem à conjuntos de dados que por metodologias matemáticas, são analisados e transformados em porcentagens.

O estudo foi realizado a partir de dados coletados no Hospital Veterinário Universitário do CCA/UNIVASF, Petrolina PE, em um projeto de extensão intitulado atendimento especializado em clínica cirúrgica. Esse projeto tinha como objetivo fornecer 
conhecimento sobre as afecções cirúrgicas aos graduandos integrantes do projeto e fornecer a sociedade um serviço de clínica e cirurgia voltados para afecções em tecidos moles, neurologia e ortopedia. Foram incluídos no levantamento, os prontuários clínicos do HVU e os arquivos radiográficos do Setor de Diagnóstico por Imagem. Todos os casos eram oriundos de atendimento pelo setor de Clínica Cirúrgica de Animais de Companhia (CCAC) do HVU. Computaram-se os casos de fratura em úmero, rádio, ulna, fêmur, tíbia e fíbula de cães e gatos, ocorridos entre o período de janeiro de 2016 a abril de 2018. Para realização do trabalho, obteve-se autorização dos responsáveis pelo hospital veterinário. Informações pessoais dos tutores não foram coletadas.

Os prontuários clínicos dos pacientes foram revistos para obtenção de cálculo das frequências. As informações registradas foram: local da fratura, idade e histórico de como ocorreu a fratura. Os dados adquiridos foram plotados no Microsoft Excel, e partir destes dados os gráficos foram gerados e analisados para posterior discussão.

\section{Resultados e Discussão}

No período de janeiro de 2016 a abril de 2018, foram registraram-se 55 casos de fratura em esqueleto apendicular, atendidos pelo setor de Clínica Cirúrgica de Animais de Companhia (CCAC) do HVU/UNIVASF. Na Figura 1 é possível visualizar que 48 destas fraturas (87\%) foram em cães, e 7 (13\%) em gatos. Milliard e Weng (2014) realizaram uma investigação semelhante no Hospital da Pardue University College of Veterinary Medicine, e observaram um total de 659 casos de fraturas traumáticas em esqueleto apendicular. Destas fraturas, 546 (83\%) ocorreram em cães e 113 (17\%) em gatos. Assim como no presente estudo, Millard \& Weng (2014) obtiveram valores percentuais maiores de fratura de esqueleto apendicular em cães quando comparados ao percentual dessas fraturas em gatos.

Scott e Mclaughlin (2006) atribuem os menores valores percentuais registrados em gatos para fraturas de esqueleto apendicular quando comparados aos cães, aos maiores cuidados que tutores de felinos demonstraram com seus animais em relação aos caninos. Destacaram também que os gatos são animais mantidos por mais tempo dentro de casa, expondo-se menos aos riscos a que os cães são submetidos Além disso, os gatos possuem vantagens ortopédicas em relação aos cães, pois têm menor peso, mais flexibilidade, ossos mais retos e menos predisposição genética para afecções ortopédicas.

Figura 1. Prevalência em porcentagem de fraturas entre cães e gatos no projeto de extensão em Petrolina - PE, entre os anos de 2016 e 2018. Abaixo é possível observar porcentagem maior de fratura em cães do que em gatos.

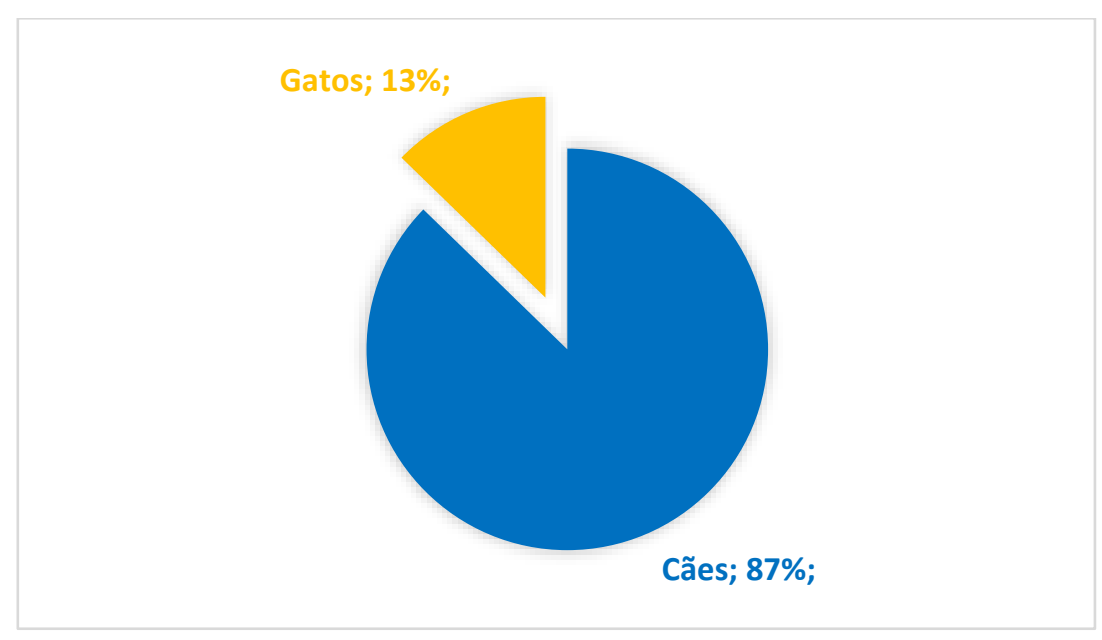

Fonte: Autores.

No que diz respeito a idade dos animais, a Tabela 1 demonstra que a maior incidência de fraturas ocorreu em animais com idade igual ou inferior a um ano. Em relação aos cães, constatou-se que, em 58\% dos casos, eles tinham idade igual ou inferior um 
ano de idade, e em $42 \%$ dos casos, tinham idade superior a um ano de idade.

Tabela 1 - Números e frequências (\%) de fraturas, por espécie e idade, de 55 casos atendidos no projeto de extensão no período de 2016 a 2018.

$\begin{array}{cccc}\text { IDADE } & \text { CÃO }(\%) & \text { GATO }(\%) & \text { TOTAL } \\ <\text { 1 ANO } & 28(58 \%) & 5(71 \%) & 33 \\ >\text { 1 ANO } & 20(42 \%) & 2(29 \%) & 22 \\ \text { TOTAL } & 48 & 7 & 55\end{array}$

Fonte: Autores.

Em estudo semelhante, realizado no Hospital Veterinário da Universidade do Norte do Paraná (UNOPAR) Kemper e Diamante (2010), computaram dados relacionados a fraturas em cães e gatos em seu setor radiográfico, no período de janeiro de 2007 até março de 2009. Constataram que 21 fraturas ocorreram em cães com menos de um ano de idade, e 9 fraturas em cães com idade superior a um ano. Um estudo de Siqueira et al. (2015) obteve 57\% das fraturas registradas em cães com menos de um ano de idade, e $32 \%$ em cães com idade superior.

No presente trabalho, as porcentagens de fraturas no que tange a idade, não se diferenciam muito em gatos quando comparadas aos cães. Observaram-se que $71 \%$ dos casos de fraturas em gatos, ocorreram naqueles com menos de um ano de idade, e 29\% nos gatos com mais de um ano de idade (Tabela 1). Dados semelhantes foram observados por Siragusi et al. (2015), em um estudo retrospectivo de 36 casos de fraturas em felinos domésticos realizado na Universidade de Marília - SP. No período de 2007 a 2014, esses autores observaram que 62\% das ocorrências de fraturas em esqueleto apendicular, foram em gatos com idade inferior a um ano e 38\% nos pacientes com idade superior. Esses números podem ser explicados pelo fato de que, a zona hipertrófica, presente na placa fisária dos ossos, ainda apresenta pouca matriz óssea, logo se torna uma região mais propicia a fraturas em cães e gatos jovens (Johnson \& Tobias, 2017). Além da presença de ossos mais frágeis, animais muito jovens apresentam pouca habilidade para evitar traumas (Vidane et al., 2014).

Em relação a etiologia das fraturas apendiculares do presente levantamento, pôde-se observar que $61 \%$ das fraturas foram decorrentes de acidentes automobilísticos e $23 \%$ resultaram de quedas. Siqueira et al. (2015) encontraram dados semelhantes, onde $52 \%$ das fraturas ocorreram devido acidentes automobilísticos e $24 \%$ foram originadas por quedas. Kemper \& Diamante (2010) relataram que 64,7\% das fraturas observadas deveram-se a acidentes automobilísticos, e 17,7\% foram consequências de quedas. Souza et al. (2011) demonstraram em seu trabalho, que 76,3\% das fraturas deveram-se a acidentes automobilísticos e 17,7\% decorreram de quedas e brigas.

Sendo assim, nota-se que nos trabalhos consultados em literatura, acidentes automobilísticos ficaram em primeiro lugar como causa de fraturas apendiculares em cães e gatos, bem como no presente trabalho. As porcentagens descritas acima para esta investigação e as demais etiologias registradas, estão presentes na Figura 2. 
Figura 2. Prevalência em porcentagem das etiologias de fraturas em esqueleto apendicular no projeto de extensão, nos anos de 2016 a 2018.

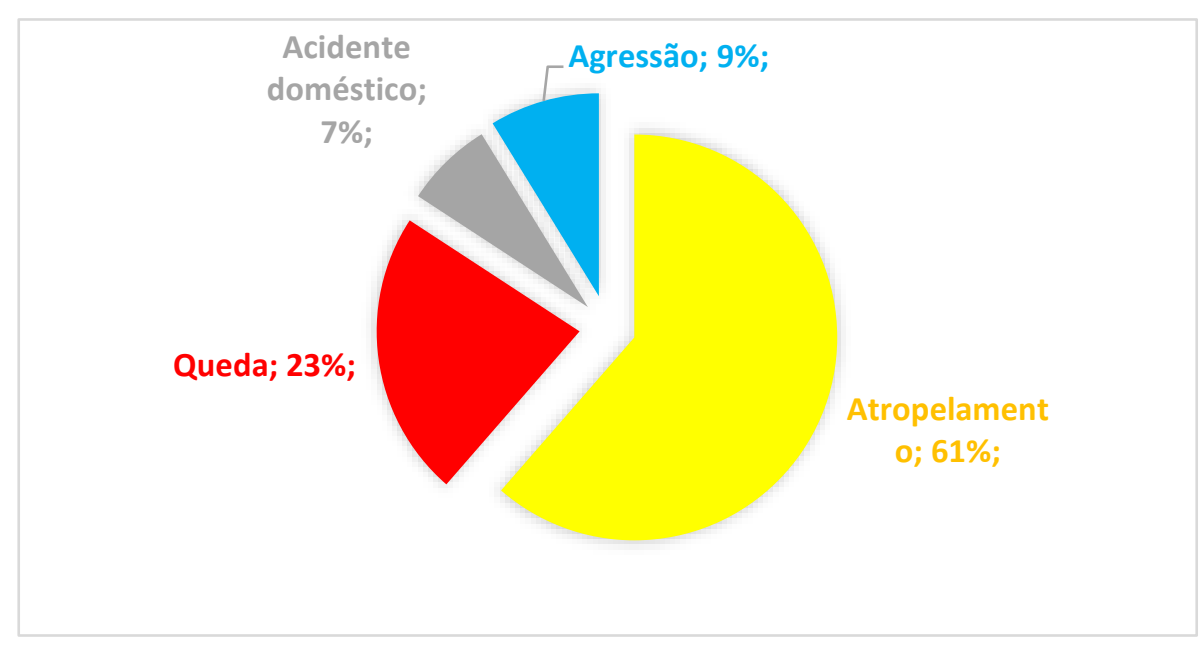

Fonte: Autores.

Libardone (2015) relataram em seu trabalho que as altas porcentagens dos traumas se devem ao grande número de cães errantes, e a negligência de alguns tutores quanto à segurança em suas residências e durante os passeios. Mendes e Arias (2012) indicam que médicos veterinários orientem os responsáveis quanto à admissão de medidas de segurança que reduzam os fatores que podem resultar em traumas. Essas medidas incluem passeios apenas com uso de guias, manter distâncias de segurança nas vias em que há trânsito de veículos, além de dar preferência a locais e horários em que o trânsito de veículos seja menor.

Esse presente estudo retrospectivo tem foco em fraturas do esqueleto apendicular, as quais foram a de maior casuística no HVU - UNIVASF. Conforme demonstra a Figura 3, do total de fraturas em esqueleto apendicular canino apresentadas nesse trabalho, o rádio e a ulna representam 19\%. Esse valor é semelhante ao encontrado por Giglio et al. (2007), que relataram que fraturas de rádio e ulna representam 20,9\% do total de casos presentes em trabalho realizado no Hospital Veterinário da Faculdade de Medicina Veterinária e Zootecnia da Universidade de São Paulo.

Valores semelhantes aos encontrados nesse presente trabalho foram relatados por Kemper e Diamante (2010) que encontraram uma percentagem de 17,64\% do total de fraturas, nos ossos rádio e ulna de cães. Siqueira et al. (2015) relataram que $41 \%$ das fraturas em esqueleto apendicular eram em rádio e ulna, um percentual muito superior aos valores encontrados para a casuística do HVU/UNIVASF no período investigado.

A pequena quantidade de tecido mole na região de rádio e ulna aumentam a probabilidade de fraturas, e estas tem mais chances de serem abertas, o que colabora para demora na cicatrização óssea. Em cães de pequeno porte a falta de tecido mole e vascularização diafisária distal em comparação com cães grandes, acabam favorecendo a alta casuística bem como casos de união retardada e não união (Fossum, 2019).

$\mathrm{Na}$ literatura consultada as fraturas umerais em cães variaram em aproximadamente $8 \%$ a $10 \%$ do total de fraturas em cães (Hill, 1977; Phillips, 1979; Johnson et al., 1994; Ness et al., 1996). No presente trabalho, o úmero representou 10\% (Figura 3) das fraturas em esqueleto apendicular de cães. Esse valor foi próximo aos encontrados por Kemper e Diamante (2010) onde 14,7\% das fraturas foram em úmero e por Siqueira et al. (2015), em que 9\% das fraturas afetaram o úmero.

Em técnica cirúrgica, a região umeral é de difícil abordagem e reparo cirúrgico, devido a sua forma em $\mathrm{S}$ com aspecto torcido, estruturas anatômicas circunvizinhas e grande densidade muscular, este último fator também pode ajudar a menores valores registrados de fratura nesse osso. Outro fator que dificulta a realização de algumas técnicas cirúrgicas, como a inserção de pinos 
intramedulares, é o afunilamento e término do canal medular que limitam o avanço do pino até o côndilo umeral (Langley-Hobbs $\&$ Straw, 2005).

Nos membros pélvicos, o fêmur foi o osso mais acometido, e correspondeu a $46 \%$ das fraturas em cães (Figura 3). Vidane et al. (2014) encontraram valores diferentes em sua casuística, na qual as fraturas em fêmur representaram 35,74\% do total. Kemper e Diamante (2010) e Siqueira et al, (2015) encontraram para a fratura de fêmur valores percentuais de $11,76 \%$ e $23 \%$ respectivamente.Decamp e Schaefer (2016) descreveram que as fraturas em fêmur correspondem de 20 a $25 \%$ de todas as fraturas do corpo animal, e $45 \%$ de todas as fraturas em ossos longos. Essas fraturas geralmente são fechadas devido a presença de grande massa muscular que protege a região.

Decamp e Schaefer (2015), descreveram que os ossos tíbia e fíbula representaram 21\% das fraturas em osso longos de cães e gatos e $11,7 \%$ das fraturas em esqueleto apendicular. Podem ser fraturas fechadas ou abertas, mas comumente são abertas, devido à pouca densidade de tecido mole ao redor da região tíbio-fibular (Palmer, 1999).

No presente trabalho, fraturas de tíbia e fíbula ficaram em segundo lugar como fratura mais comum no esqueleto apendicular de cães, e correspondeu a 25\% (Figura 3) do total de fratura apresentado em cães. Alguns trabalhos encontraram valores semelhantes para fraturas nestes ossos em cães: Vidane et al. (2014), Siqueira et al. (2015) relataram 21,92\% e 26\% de fraturas nestes ossos, respectivamente. Enquanto Kemper e Diamante (2010) observaram menor percentual, (11,76\%) em sua casuística.

Figura 3. Representação gráfica do percentual de ossos fraturados em cães no projeto de extensão.

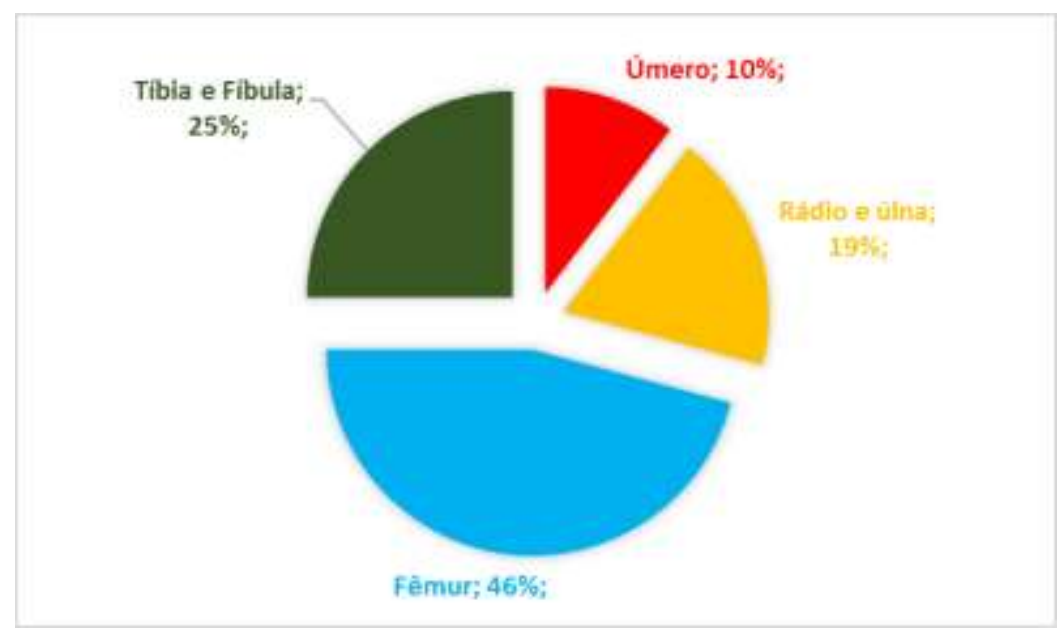

Fonte: Autores.

Alguns trabalhos semelhantes a este descreveram que 5\% a 13\% das fraturas em gatos, ocorreram no úmero (Hill, 1977; Phillips, 1979; Johnson et al., 1994; Ness et al., 1996). A Figura 4 demonstra que $43 \%$ das fraturas relatadas em gatos foram observadas em úmero. Valores esses muito diferentes dos encontrados por Siragusi et al. (2015) que demonstraram em seu trabalho um percentual ainda menor (3\%), de fraturas no úmero de gatos. Fraturas proximais em úmero são incomuns em felinos, mas quando acontecem, corriqueiramente envolvem o grande tubérculo e a cabeça do úmero. Esse tipo de fratura é mais comum em gatos jovens que ainda apresentam a linha fisária aberta (Scott \& Mclaughlin, 2006).

Os gatos, assim como os cães, apresentaram maior percentual de fratura em região femoral. Pode-se observar que 57\% das fraturas em gatos foram em fêmur (Figura 4). Vidane et al. (2014), em seu estudo realizado em três unidades de atendimento veterinário na cidade de Maputo - Moçambique, encontraram um percentual de 35,14\% do total das fraturas em fêmur de gatos. Siragusi et al. (2015) relataram valor aproximado ao do presente trabalho, onde as fraturas em fêmur de gatos, representaram $48 \%$ do total de fratura analisados. 
Figura 4. Representação gráfica do percentual de ossos fraturados em gatos no projeto de extensão.

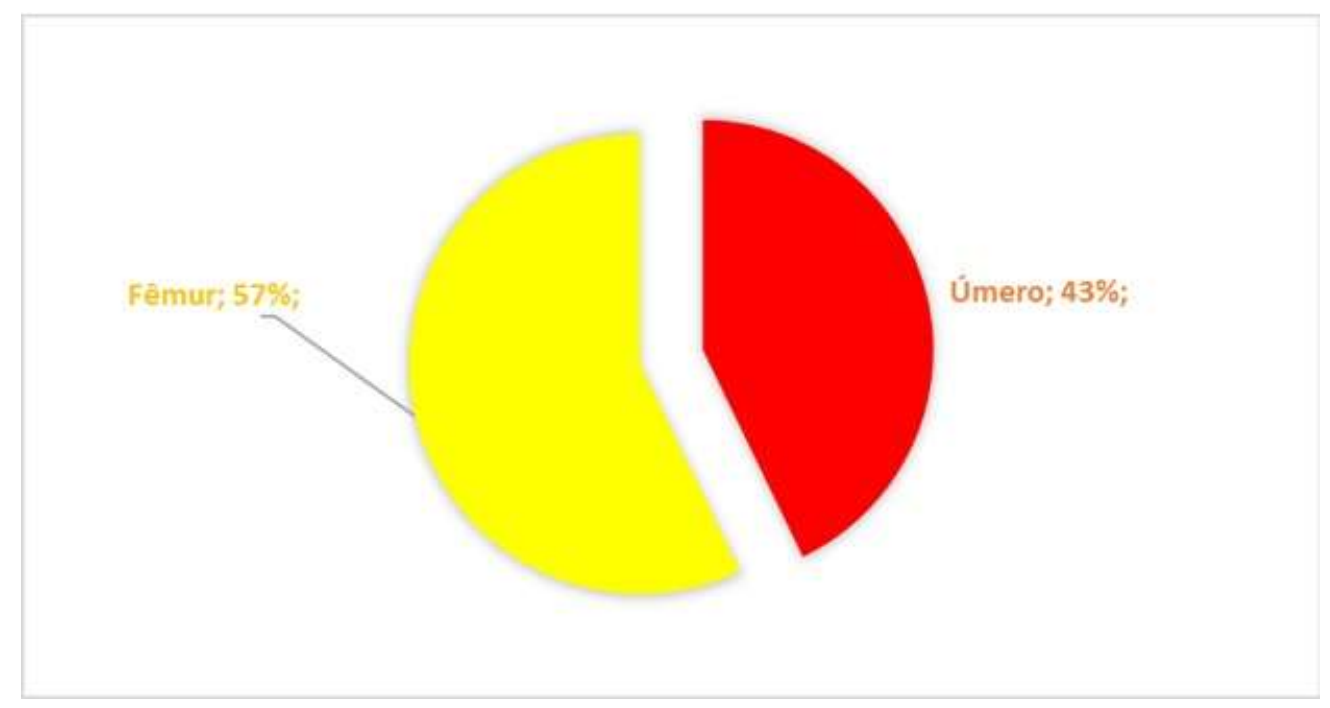

Fonte: Autores.

Valores para rádio, ulna, tíbia e fíbula não puderam ser analisados, para felinos, pois não alcançaram pontuação.

Estudos epidemiológicos que investigam a prevalência de fraturas e as causas atreladas as mesmas, têm aumentado nas últimas décadas (Kemper \& Diamante, 2010). O conhecimento da casuística de fraturas em esqueleto apendicular de cães e gatos, bem como de fatores etiológicos, na cidade de Petrolina-Brasil, têm sua importância a fim trazer a tona os principais fatores de riscos aos pacientes, bem como auxilia na elaboração de medidas de controle e propagação de educação por meio de medidas preventivas divulgadas aos tutores. Além disso contribui de forma mais abrangente, como fonte epidemiológica para outras investigações semelhantes, o que agrega mais embasamento aos levantamentos sobre prevalência de fraturas em cães e gatos.

\section{Conclusão}

Baseado nas informações obtidas, foi possível observar que a maior casuística de fraturas, em esqueleto apendicular, atendidas no Hospital Veterinário da UNIVASF, no período de análise, se deram em cães. Um fator importante a se considerar foi a idade dos animais acometidos, que em sua maioria, tinham idade igual ou inferior a um ano, reforçando o fato de que até um ano de idade existe uma fragilidade óssea que facilita o acontecimento da fratura. O osso mais acometido tanto em cães como em gatos foi o fêmur. Entre as causas das fraturas, o acidente automobilístico ficou em primeiro lugar, corroborando com os resultados das demais literaturas consultadas. Uma significativa parcela de animais errantes e a falta de orientação aos tutores sobre medidas de segurança em passeios contribuem para esses valores encontrados.

Este tipo de estudo contribui para que a partir dos dados contabilizados de uma casuística de fraturas, possamos entender quais os fatores que podem estar implicados na sua etiologia, e melhorar o atendimento a esses pacientes, uma vez que podemos orientar os tutores baseado, também, em dados locais.

Devido a vigência do projeto de extensão, o presente estudo não pode se extender por mais de 2 anos. Em trabalhos futuros sugere-se avaliação de longos periodos, entre dez e quinze anos para melhor contabilização de dados.

\section{Agradecimentos}

Agradeço a Deus, minha família, meu orientador, supervisora e colegas que participaram dos procedimentos e pelo suporte durante a contrução desse trabalho. 


\section{Referências}

Beale, B. (2004). Orthopedic clinical techniques femur fracture repair. Clinical Techniques in Small Animal Practice, 19(3), 134-150.

Corr, S. (2012). Complex and open fractures: a straightforward approach to management in the cat. Journal of feline medicine and surgery, 14(1), 55-64.

DeCamp, C. E. (2015). Brinker, Piermattei and Flo's handbook of small animal orthopedics and fracture repair. Elsevier Health Sciences.

Doblaré, M., Garcı, J. M., \& Gómez, M. J. (2004). Modelling bone tissue fracture and healing: a review. Engineering Fracture Mechanics, 71(13-14), 1809-1840.

Giglio, R. F., de Almeida Sterman, F., de Campos Fonseca, A. C. B., Unruh, S. M., Schmaedecke, A., \& Ferringno, C. R. A. (2007). Estudo retrospectivo de radiografias com fraturas rádio e ulna em cães. Brazilian Journal of Veterinary Research and Animal Science, 44, $122-124$.

Gustilo, R. B.; \& Anderson, J. T. (1976). Prevention of Infection in the Treatment of One Thousand and Twenty-five Open Fractures of Long Bones. J Bone Joint Surg Am, 58, 453-458.

Hayashi, K., Schulz, K. S., \& Fossum Terry, W. (2019). Principles of Fracture Diagnoses and Management. Textbook of Small Animal Surgery. Fossum TW, 5th Ed Elsevier Health Sciences Missouri, 976-1025.

Harasen, G. (2003a). Common long bone fractures in small animal practice—part 1. The Canadian Veterinary Journal, 44(4), 333.

Harasen, G. (2003b). Common long bone fracture in small animal practice-Part 2. The Canadian Veterinary Journal, 44(6), 503.

Hill, F. W. G. (1977). A survey of bone fractures in the cat. Journal of Small Animal Practice, 18(7), 457-463.

Hulse, D., \& Hyman, B. (2003). Biomecânica e biologia das fraturas. SLATTER, D. Manual de cirurgia de pequenos animais, 3, $1785-1792$.

Johnson, J. A., Austin, C., \& Breur, G. J. (1994). Incidence of canine appendicular musculoskeletal disorders in 16 veterinary teaching hospitals from 1980 through 1989. Veterinary and Comparative Orthopaedics and Traumatology, 7(02), 56-69.

Johnston, S. A., \& Tobias, K. M. (2017). Veterinary Surgery: Small Animal Expert Consult-E-Book: 2-Volume Set. Elsevier Health Sciences.

Kemper, B., \& Diamante, G. A. C. (2010). Estudo retrospectivo das fraturas do esqueleto apendicular de cães atendidos no Hospital Veterinário da Universidade Norte do Paraná (UNOPAR) no período de janeiro de 2007 a março de 2009. Journal of Health Sciences, 12(2).

Kumar, K., Mogha, I. V., Aithal, H. P., Kinjavdekar, P., Amarpal, Singh, G. R., \& Kushwaha, R. B. (2007). Occurrence and pattern of long bone fractures in growing dogs with normal and osteopenic bones. Journal of Veterinary Medicine Series A, 54(9), 484-490.

Langley-Hobbs, S. J., \& Straw, M. (2005). The feline humerus. Veterinary and Comparative Orthopaedics and Traumatology, 18(01), 01-06.

Libardoni, R. D. N., Serafini, G. M. C., Oliveira, C. D., Schimites, P. I., Chaves, R. O., Feranti, J. P. S., \& Soares, A. V. (2016). Appendicular fractures of traumatic etiology in dogs: 955 cases (2004-2013). Ciência Rural, 46(3), 542-546.

Marretta, S. M., \& Schrader, S. C. (1983). Physeal injuries in the dog: a review of 135 cases. Journal of the American Veterinary Medical Association.

Mendes, D. S., \& Arias, M. V. B. (2012). Traumatismo da medula espinhal em cães e gatos: estudo prospectivo de 57 casos. Pesquisa Veterinária Brasileira, 32 (12), 1304-1312.

Millard, R. P., \& Weng, H. Y. (2014). Proportion of and risk factors for open fractures of the appendicular skeleton in dogs and cats. Journal of the American Veterinary Medical Association, 245(6), 663-668.

Ness, M. G., Abercromby, R. H., May, C., Turner, B. M., \& Carmichael, S. (1996). A survey of orthopaedic conditions in small animal veterinary practice in Britain. Veterinary and Comparative Orthopaedics and Traumatology, 9(02), 43-52.

Palmer, R. H. (1999). Biological osteosynthesis. Veterinary clinics: small animal practice, 29(5), 1171-1185.

Pereira, A. S., Shitsuka, D. M., Parreira, F. J., \& Shitsuka, R. (2018). Metodologia da pesquisa científica.

Phillips, I. R. (1979). A survey of bone fractures in the dog and cat. Journal of Small Animal Practice, 20(11), 661-674.

Pozzi, A., \& Lewis, D. D. (2009). Surgical approaches for minimally invasive plate osteosynthesis in dogs. Veterinary and Comparative Orthopaedics and Traumatology, 22(04), 316-320.

Radasch, R. M. (1999). Biomechanics of bone and fractures. Veterinary Clinics of North America: Small Animal Practice, 29(5), 1045-1082.

Rosol, T. J., \& Capen, C. C. (1997). Calcium-regulating hormones and diseases of abnormal mineral (calcium, phosphorus, magnesium) metabolism. In Clinical biochemistry of domestic animals (pp. 619-702). Academic press.

Roush, J. K. (2005). Management of fractures in small animals. Veterinary Clinics: Small Animal Practice, 35(5), $1137-1154$.

Schmökel, H. G., Stein, S., Radke, H., Hurter, K., \& Schawalder, P. (2007). Treatment of tibial fractures with plates using minimally invasive percutaneous osteosynthesis in dogs and cats. Journal of small animal practice, 48(3), 157-160.

Scott, H. (2005). Repair of long bone fractures in cats. In Practice, 27(8), 390-397.

Scott, H., \& McLaughlin, R. (2006). Feline orthopedics. CRC Press. 
Shahar, R., Banks-Sills, L., \& Eliasy, R. (2003). Mechanics of the canine femur with two types of hip replacement stems. Veterinary and Comparative Orthopaedics and Traumatology, 16(03), 145-52.

Simpson, A. M. (2004). Fractures of the humerus. Clinical techniques in small animal practice, 19(3), $120-127$.

Siqueira1, R. C., Siragusi, R. H., Scorsato, M. F., Souza, J. B., \& Franco, R. P. (20015). Estudo retrospectivo da ocorrência de fraturas em ossos longos nos cães atendidos durante o período de 2006 a 2013 na universidade de Marília-SP/Brasil Retrospective study of fractures in long bones in dogs assisted during the period of 2006-2013 at the University of Marília-SP/Brazil.

Siragusi, R. H., Siqueira, R. C., \& Franco, R. P. (2015). Estudo retrospectivo das fraturas em felinos atendidos no Hospital Veterinário da Universidade de MaríliaSP/Brasil no Período de 2007 a 2014. Revista de Educação Continuada em Medicina Veterinária e Zootecnia do CRMV-SP, 13(2), 10-15.

Souza, M. M. D. D., Rahal, S. C., Padovani, C. R., Mamprim, M. J., \& Cavini, J. H. (2011). Afecções ortopédicas dos membros pélvicos em cães: estudo retrospectivo. Ciência Rural, 41(5), 852-857.

Sumner-Smith, G., \& Cawley, A. J. (1970). Nonunion of fractures in the dog. Journal of Small Animal Practice, 11, $311-325$.

Tillson, D. M. (1995). Open fracture management. Veterinary Clinics: Small Animal Practice, 25(5), 1093-1110.

Thrall, D. E. (2019). Diagnóstico de radiologia veterinária. Elsevier Editora Ltda. 287p.

Toombs, J. P., Wallace, L. J., Bjorling, D. E., \& Rowland, G. N. (1985). Evaluation of Key's hypothesis in the feline tibia: an experimental model for augmented bone healing studies. American journal of veterinary research, 46(2), 513-518.

Unger, M., Montavon, P. M., \& Heim, U. F. A. (1990). Classification of fractures of long bones in the dog and cat: introduction and clinical application. Veterinary and Comparative Orthopaedics and Traumatology, 3(02), 41-50.

Vashishth, D. (2008). Small animal bone biomechanics. 794-797.

Vaughan, L. C. (1964). A clinical study of non-union fractures in the dog. Journal of Small Animal Practice, 5(2), 173-177

Vidane, A. S., Elias, M. Z. J., Cardoso, J. M. M., Come, J. A. S. S., Harun, M., \& Ambrósio, C. E. (2014). Incidência de fraturas em cães e gatos da cidade de Maputo (Moçambique) no período de 1998-2008. Ciência Animal Brasileira, 15(4), 490-494.

Welch, J. A., Boudrieau, R. J., Dejardin, L. M., \& Spodnick, G. J. (1997). The intraosseous blood supply of the canine radius: implications for healing of distal fractures in small dogs. Veterinary Surgery, 26(1), 57-61. 